\section{Total Intestinal Aganglionosis Presenting as Duodenal Obstruction}

In the majority of cases of Hirschsprung's disease, aganglionosis is limited to the rectum and lower sigmoid colon. Longer segments of the colon or the entire colon may be involved in some cases, the latter often with partial involvement of the small intestine. When there is this degree of aganglionosis, differentiation from other causes of neonatal intestinal obstruction may be difficult.

Six cases of total colonic with partial small intestinal aganglionosis have been seen in Manchester in the past 20 years (S. Ahmed, unpublished). In this, the seventh case, aganglionosis extended from the anal canal to the duodenum, rendering differentiation from other causes of neonatal duodenal obstruction impossible. The correct diagnosis was suspected at the third laparotomy and confirmed at necropsy.

\section{Case Report}

A female born at term, weighing $3.9 \mathrm{~kg}$, was admitted at 24 hours of age with bile-stained vomiting and abdominal distension. She had passed meconium spontaneously. Examination revealed a distended but soft abdomen with normal bowel sounds. Rectal examination was normal and was followed by profuse passage of meconium.

She was kept under observation but continued vomiting. An upper gastrointestinal series, performed at the age of 6 days, showed a partial duodenal obstruction (Fig.). At operation there was malrotation of the intestines and the first and second parts of the duodenum were dilated and tapered into the third part. Two tight, separated bands were running across the third part, and complete Ladd's procedure was performed.

Five days later there was still a large aspirate of bile from the stomach and further $x$-rays showed that the duodenum was still obstructed. At the second laparotomy duodenoduodenostomy and gastrostomy were performed, a transanastomotic feeding tube being left in situ.

There was no improvement after this operation and the child continued to lose a lot of bile. One week later she developed a duodenal fistula, and at reoperation the anastomosis was seen to have come apart completely. It was repaired and this time a gastrojejunostomy was performed. At this stage it was suspected that the child might be suffering from extensive Hirschsprung's disease. The appendix was therefore removed for histological examination which showed absence of ganglion cells and nerve fibres as well. The child died a few days later.

At necropsy the small bowel was found to have a universal mesentery and there was peritonitis. Detailed histological examination showed that there were normal ganglion cells in the oesophagus, stomach, and pylorus. Examination of the rectum, colon, appendix, ileum, and jejunum revealed the complete absence of ganglion cells and nerve fibres. It was difficult to interpret the sections made from the most proximal jejunum (the area of surgical reconstruction), but the evidence was that ganglion cells were absent in this region, as well as in the duodenum.

Family history. The patient was the only child of healthy parents. There was no family history of histologically proven Hirschsprung's disease but her maternal aunt had died from neonatal intestinal obstruction in 1945. Whether she had Hirschsprung's disease or not, is a matter of conjecture.

\section{Discussion}

Bodian, Carter, and Ward (1951), reporting a case of total intestinal aganglionosis in a male child, described the condition as agenesis of intramural plexuses and commented on the widespread absence of ganglion cells and nerve fibres as well. Bodian and Carter (1963) reported a second case of aganglionosis from the duodenum to the anal canal in a female sib of the first patient, in whom an identical histological picture was present. Boggs and Kidd (1958) reported another two sibs (one male and one female) with absence of innervation of the jejunum, ileum, and colon. One case of total intestinal aganglionosis was reported byt Lee in 1955 (sex not known), and another by Walker, Kempson, and Ternberg in 1966 (male). Thus, the case reported here is the seventh case of total intestinal aganglionosis in the English literature.

The male-to-female ratio in these 7 cases $(3$ male 3 female, 1 not known) is similar to that seen in most series of long segment Hirschsprung's disease, in contrast to a $4: 1$ ratio in the commoner rectosigmoid form of the disease (Coran et al., 1969). There also appears to be a high risk of future sibs being affected.

The histological appearance in our case was similar to that first described by Bodian et al. (1951) and also observed by Boggs and Kidd (1958). This histological characteristic of absence of both ganglion cells and nerve fibres appears to be peculiar to cases where there is total intestinal aganglionosis; but we have also seen it in patients with total colonic and partial small intestinal aganglionosis though not in the commoner rectosigmoid form of Hirschsprung's disease (S. Ahmed, unpublished).

In most patients with total intestinal aganglionosis the clinical presentation was that of an intestinal disorder with failure to pass meconium. In contrast, in our case the baby passed a surprisingly 
normal amount of meconium in the first few days of life, with fairly diffuse distribution of gas on earlier $x$-ray films (Fig.). Signs of duodenal obstruction with paucity of gas distally became evident on later films. Not surprisingly, the operative manoeuvres were aimed at the duodenum, whereas in the previously reported cases the common operation performed was either a colostomy or an ileostomy.

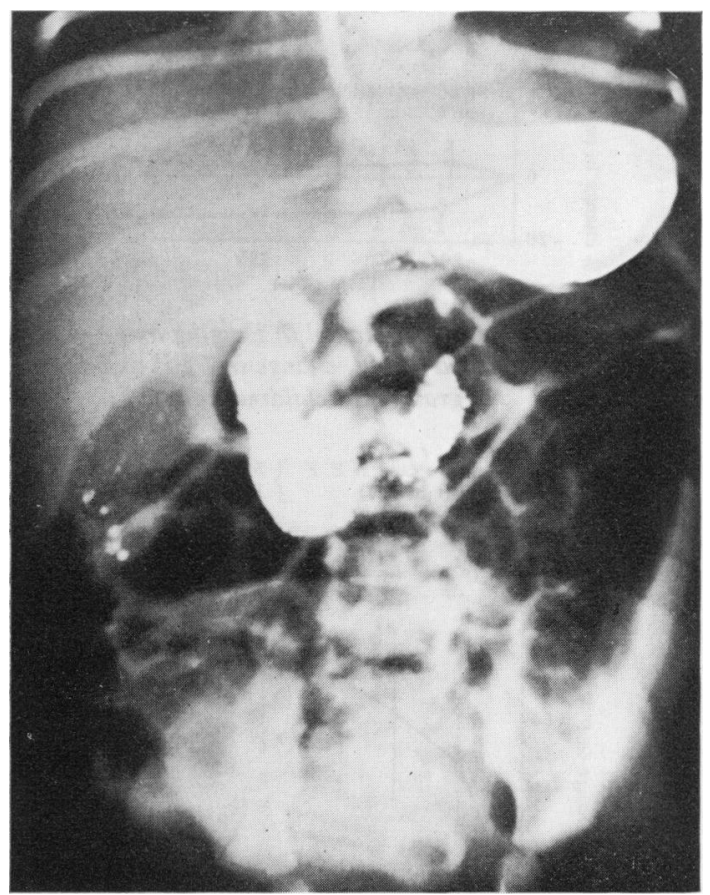

FIG.-Lipiodol was inserted into the stomach via a nasogastric tube. The contrast medium was seen to be held up at the third part of the duodenum, though abundant gas was seen in the distal gastrointestinal tract.

Malrotation of the intestines has been reported previously in association with Hirschsprung's disease (Rickham and Johnston, 1969) and has been seen in other cases of Hirschsprung's disease in Manchester (S. Ahmed, unpublished). The presence of malrotation complicated the clinical picture in this case. It appears that in cases of malrotation or when the exact nature of the duodenal obstruction is not clear, the appendix should be taken out for urgent histological examination. If Hirschsprung's disease is diagnosed, further serial biopsies of the ileum and jejunum should always be undertaken as location of the cone area is notoriously difficult in such cases. The degree of involvement of the small bowel would thus be elucidated, enabling one to perform the appropriate surgical procedure. On the other hand, knowledge of the presence of total intestinal aganglionosis would contraindicate further surgical measures, the condition being incompatible with life.

\section{Summary}

A case of total intestinal aganglionosis is reported. The patient presented as a duodenal obstruction with intestinal malrotation. The reason for the failure of operative manoeuvres to relieve the obstruction was suspected before the patient died, and confirmed on histological studies which showed absence of ganglion cells and nerve fibres from the anal canal to the duodenum.

We would like to thank Dr. John Fawcitt for radiological comments and Miss Perry for the illustration.

\section{REFERENCES}

Bodian, M., and Carter, C. O. (1963). A family study of Hirschsprung's disease. Annals of Human Genetics, 26, 261.

Bodian, M., Carter, C. O., and Ward, B. C. H. (1951). Hirschsprung's disease. Lancet, 1, 302.

Boggs, J. D., and Kidd, J. M. (1958). Congenital abnormalities of intestinal innervation. Pediatrics, 21, 261.

Coran, A. G., Biordal, R., Eek, S., and Knutrud, O. (1969). The surgical management of total colonic and partial small intestinal aganglionosis. Fournal of Pediatric Surgery, 4, 531.

Lee, C. M., Jr. (1955). Megacolon, with particular reference to Hirschsprung's disease. Surgery, 37, 762.

Rickham, P. P., and Johnston, J. H. (1969). Hirschsprung's Disease. In Neonatal Surgery, p. 382. Butterworths, London.

Walker, A. W., Kempson, R. L., and Ternberg, J. L. (1966). Aganglionosis of the small intestine. Surgery, 60, 449.

S. AHMED, * S. J. CoHEN, and S. I. JACOBS†

Booth Hall Children's Hospital, Manchester.

^Correspondence to Dr. S. Ahmed, Royal Manchester Children's Hospital, Pendlebury, Manchester M27 1HA.

tNow at St. James's Hospital, Leeds.

\section{Dosage of Oral Salbutamol in Asthmatic Children}

Salbutamol has been shown to be a potent bronchodilator with less cardiac action than isoprenaline (Warrell et al., 1970; Kamburoff and Prime, 1970) or orciprenaline (Riding, Dinda, and Chatterjee, 1970). This is thought to be due to its selective activity on $\beta-2$ adrenergic receptors in bronchial muscle (Cullum et al., 1969). The optimal dose for use clinically should reduce airways resistance without causing side effects, particularly tachycardia. Therefore a dose response study was undertaken to select a dose of oral salbutamol which would give maximal relief to a child with 